\title{
Aceros aluminotérmicos. Nuevas aplicaciones ${ }^{(\bullet)}$

\author{
J.M. Duart Blay*, J.A. Pero-Sanz Elorz y L.F. Verdeja González ${ }^{* * *}$
}

Resumen

La metalotermia es un proceso físicoquímico basado en la posibilidad de reducción de óxidos metálicos (hierro, cromo, manganeso, cobre) por metales altamente afines por el oxígeno, como son aluminio, magnesio y titanio. La reacción es altamente exotérmica dando lugar a un caldo metálico separado de su escoria, por un proceso de reducción y afino combinados. La patente tecnológica, desarrollada inicialmente por Thermit y perfeccionada por otras Compañías, lleva en vigor más de 50 años; se aplica hoy, casi exclusivamente, a la obtención de aceros y cobres para uniones soldadas complejas. Particular aplicación o interés presentan en la tecnología de los ferrocarriles para la obtención del carril continuo, prácticamente implantado en todo el mundo y en soldaduras cable de cobre-carril de acero empleadas en las señalizaciones para control de tráfico. En este trabajo se aportan las bases termodinámicas de la aluminotermia del hierro y su aplicación a la soldadura compleja de cruzamientos, juntas de dilatación y desvíos en FF.CC., que combinan aceros Hadfield, aceros inoxidables y aceros perlíticos de diferentes propiedades mecánicas. Las uniones deben ser compactas, resistentes y duras en los niveles que se citan en el trabajo, según requisitos exigidos por la circulación en líneas de alta velocidad $(350 \mathrm{~km} / \mathrm{h}$.), actualmente en construcción en España, pero que resultan generalizables a otro tipo de líneas menos exigentes y a ferrocarriles mineros.

Palabras clave Aceros aluminotérmicos. Soldadura. Propiedades mecánicas.

\section{Aluminothermics steels. News applications}

Abstract

Keywords
The metallothermy is a process based on physical - chemistry possibility of metallic oxides reduction $(\mathrm{Fe}, \mathrm{Cr}, \mathrm{Mn}, \mathrm{Cu}$ ) for oxygen strong affinity metals, like $\mathrm{Al}, \mathrm{Mg}$ and $\mathrm{Ti}$. The reaction is highly exothermic giving place to a metallic phase and slag separation by refining and reduction process simultaneously. The technological developed patent initially by Thermit - and improved for other Companies - works well by more than 50 years; today it is employed almost exclusively for obtaining steels and coppers for complex welded joints unions. Particular application or interest presents in the Railways technology for building continuous tracks, practically implanted in all the word and in welding copper cable rope - steel rail employed for traffic signals control. This work displays the fundamental thermodynamic principles of the aluminothermics of iron and their application to the complex weld of crossrail, dilation joints and bypass in Railways, that combine Hadfield steels, stainless steels and pearlitic steels of different mechanical properties. The unions should be compact, with strength and hardness in the levels that are cited in the work, according to requirements demanded by high speed $(350 \mathrm{~km} / \mathrm{h}$.) lines, now in construction in Spain, but they result of general use to whatever class of less demanding lines and to mining railroads.

Aluminothermics steels. Welding. Mechanical properties.

(•) Trabajo recibido el día 22 de abril de 2003 y aceptado en su forma final el día 9 de enero de 2004.

(*) División de Soldadura Aluminotérmica, KLK Electromateriales. Apartado 333, 33280 Gijón, E-mail:j.cueli@klk.es

(**) E.T.S.I. de Minas de Madrid. Dpto. de Materiales. Universidad Politécnica de Madrid. Spain, Rios Rosas 21. Madrid 28003. E-mail: jperosanz@dim.upm.es.

${ }^{* * *}$ E.T.S.I. de Minas de Oviedo, Dpto. de Ciencia de los Materiales e Ingeniería Metalúrgica, Universidad de Oviedo, Spain, Independencia 13, Oviedo 33004, E-mail: Ifv@etsimo.uniovi.es. 


\section{INTRODUCCIÓN}

Desde la aparición del ferrocarril, se han realizado muchos esfuerzos para incrementar la longitud de los tramos continuos de vías y reducir los problemas asociados a las discontinuidades entre los mismos (uniones roblonadas con un vano entre carriles). Durante varias décadas, la posibilidad de vía continua pareció impracticable por las tensiones longitudinales generadas en los carriles por los cambios de temperatura. Se empleaban carriles de 12 o $18 \mathrm{~m}$ de longitud, embridados mecánicamente, con el suficiente número de puntas (celas) para eliminar los referidos esfuerzos. El empleo del carril continuo, soldado, comenzó en los años 50, unido al empleo de traviesas y sujeciones más rígidas y un balasto adecuado. Carriles de 18/36 m de longitud se sueldan eléctricamente en instalaciones fijas, hasta constituir tramos continuos, por ejemplo de 144/288 m, que, posteriormente a su transporte con se sueldan aluminotérmicamente, in situ, obteniéndose la "vía continua". Las ventajas que se derivan -mayor velocidad y menor consumo energético de los trenes, mantenimiento más sencillo, confort y seguridad de los viajeros- son evidentes. Por otro lado, las ventajas de la soldadura aluminotérmica respecto a la eléctrica, su directa competidora, son las siguientes ${ }^{[1}$ y 2$]$ :

- Bajo coste de equipos y materias primas

- No requiere ajuste de energía eléctrica

- La formación de soldaduras puede realizarse en pocas semanas

- Permite soldar carriles normales, duros y extraduros (aceros al carbono o débilmente aleados) cualquiera que sea su geometría en variedad de formas o perfiles y, también, aceros Hadfield ( $1 \% \mathrm{C}-13 \% \mathrm{Mn}$ ) para cruzamiento y vías de escape $^{[3]}$.

En la Nota Técnica presente, se describe el producto aluminotérmico (kit aluminotérmico) cuya caracterización pormenorizada está cubierta, ciertamente, por patente. Abundar en detalles, tal y como podía ser la simulación por FEM (Modelo por Elementos Finitos) $)^{[4]}$, no sería procedente ni admisible por la empresa KLK-ELECTRO MATRIALES, propietaria del know-how y tecnología de proceso.

El acero aluminotérmico de KLK-SOLDAL (del grupo KLK-ELECTROMATRIALES) se dedica fundamentalmente a la exportación (RENFE-GIF es un cliente más), en un $80 \%$, aproximadamente, en muy dura y a veces no leal competencia con las dos empresas multinacionales - ELETROTHERMIT y RAILTECH - que hasta hace menos de diez años se repartían el mercado internacional.

\section{LA REACCIÓN ALUMINOTÉRMICA}

Los fundamentos termodinámicos de la reducción pirometalúrgica de los óxidos metálicos se encuentran recogidos en los diagramas de Ellingham ${ }^{[5}$ y 6$]$. Cualquier metal, con una energía libre estándar de formación, $\Delta_{\mathrm{f}} \mathrm{G}^{\circ}(\mathrm{T})$, para sus formulaciones oxidadas determinadas, puede actuar de reductor de todos aquellos óxidos que tengan una $\Delta_{\mathrm{f}} \mathrm{G}^{\circ}(\mathrm{T})$ superior.

Entre los reductores más usuales se encuentran los gases $\left(\mathrm{CH}_{4}, \mathrm{H}_{2}, \mathrm{CO}\right)$, el cok y los metales activos (aquellos que posean una $\Delta_{\mathrm{f}} \mathrm{G}^{\circ}(\mathrm{T})$ en Ellingham muy negativa).

Los metales activos son agentes reductores indispensables cuando se quiere obtener un producto libre de contaminaciones carbonosas o nitruradas. En algunos casos, constituye la única alternativa para alcanzar calidad metalúrgica y propiedades mecánicas adecuadas (metalurgia del titanio).

No obstante, la utilización de los metales activos como agentes reductores (introducciones metalotérmicas) puede tener otras importantes aplicaciones derivadas del calor de reacción intercambiado durante el proceso. El calor cedido por las reacciones metalotérmicas puede utilizarse como fuente de energía química capaz de producir la fusión y, por tanto, la soldadura fuerte de los aceros.

Por otra parte, la temperatura que se precisa alcanzar en los productos de la reacción aluminotérmica estará supeditada por el punto de fusión de la alúmina, $\mathrm{Al}_{2} \mathrm{O}_{3}\left(2.054^{\circ} \mathrm{C}\right)$. La temperatura que se alcance en los productos como resultado de la reacción aluminotérmica ha de ser tal que no sólo debe permitir la fusión del acero sino también de la alúmina. Es preciso garantizar que ninguna partícula de óxido de aluminio (inclusión) quede infiltrada en el fundido, por lo que es conveniente que el $\mathrm{Al}_{2} \mathrm{O}_{3}$ se encuentre en estado líquido.

Las reacciones de reducción aluminotérmica para cada uno de los óxidos del sistema $\mathrm{Fe}-\mathrm{O}$ son las siguientes:

$$
\begin{array}{rr}
1 / 2 \mathrm{Fe}_{2} \mathrm{O}_{3}(\mathrm{~s})+\mathrm{Al}(\mathrm{s}) & \mathrm{Fe}(\mathrm{I})+1 / 2 \mathrm{Al}_{2} \mathrm{O}_{3} \\
1 / 3 \mathrm{Fe}_{3} \mathrm{O}_{4}(\mathrm{~s})+8 / 9 \mathrm{Al}(\mathrm{s}) & \mathrm{Fe}(\mathrm{I})+4 / 9 \mathrm{Al}_{2} \mathrm{O}_{3}(\mathrm{I}) \\
\mathrm{FeO}(\mathrm{s})+2 / 3 \mathrm{Al}(\mathrm{s}) & \mathrm{Fe}(\mathrm{I})+1 / 3 \mathrm{Al}_{2} \mathrm{O}_{3}(\mathrm{I})
\end{array}
$$


Es decir, que para obtener una misma cantidad de soldadura (un átomo-gramo de $\mathrm{Fe}, 55,85 \mathrm{~g}$ de hierro) se necesita utilizar:

a) Un átomo-gramo de aluminio ( $27 \mathrm{~g}$ de $\mathrm{Al}$ ), si se parte de $\mathrm{Fe}_{2} \mathrm{O}_{3}$.

b) $8 / 9$ átomos-gramo de aluminio ( $24 \mathrm{~g}$ de $\mathrm{Al}$ ), si se trata del $\mathrm{Fe}_{3} \mathrm{O}_{4}$.

c) $2 / 3$ átomos-gramo de aluminio (18 g de $\mathrm{Al}$ ), si se utiliza el $\mathrm{FeO}$ (cascarilla de laminación).

Por otra parte, empleando el módulo del balance de materia y energía del programa HSC Chemistry 3.02 de Outokumpu, se pueden calcular las diferentes temperaturas adiabáticas para la reacción aluminotérmica, según que la materia prima del sistema $\mathrm{Fe}-\mathrm{O}$ sea el $\mathrm{Fe}_{2} \mathrm{O}_{3}$, el $\mathrm{Fe}_{3} \mathrm{O}_{4}$ o el $\mathrm{FeO}$. En la tabla I, se muestran los resultados de la temperatura adiabática de reacción aluminotérmica del $\mathrm{Fe}_{2} \mathrm{O}_{3}, \mathrm{Fe}_{3} \mathrm{O}_{4}$ y $\mathrm{FeO}$, que se identifica con aquella temperatura que podría alcanzarse en el sistema si las pérdidas de calor por conducción, convección y radiación fueran nulas.

La utilización del hematites, $\mathrm{Fe}_{2} \mathrm{O}_{3}$, conlleva una temperatura adiabática de reacción tan elevada que, incluso, puede superar a la de ebullición del hierro $\left(3.070^{\circ} \mathrm{C}\right)$. Se prefiere trabajar con $\mathrm{FeO}$ que, además de presentar un menor consumo de aluminio, tiene una temperatura adiabática de reacción lo suficientemente alta como para permitir la fusión de los productos de la reacción ( $\mathrm{Fe}$ y $\mathrm{Al}_{2} \mathrm{O}_{3}$ ).

\section{PROCESOS DE SOLDADURA MÁS USUALES. TECNOLOGÍA "KLK-SOLDAL"}

Los kits de soldadura KLK-SOLDAL está formados por una carga aluminotérmica, un dispositivo de destape y el conjunto molde-placa de base-tapón (Fig. 1). Las cargas aluminotérmicas están formadas por una mezcla de granallas de óxido de hierro,

Tabla I. Temperaturas adiabáticas de reacción para la aluminotermia de los óxidos de hierro

Table I. Adiabatic temperatures of reaction for the aluminothermic of iron oxide

\begin{tabular}{cc}
\hline Óxido & Temperatura $\left({ }^{\circ} \mathrm{C}\right)$ \\
\hline $\mathrm{Fe}_{2} \mathrm{O}_{3}$ & 3.266 \\
$\mathrm{Fe}_{3} \mathrm{O}_{4}$ & 3.057 \\
$\mathrm{FeO}$ & 2.814 \\
\hline
\end{tabular}

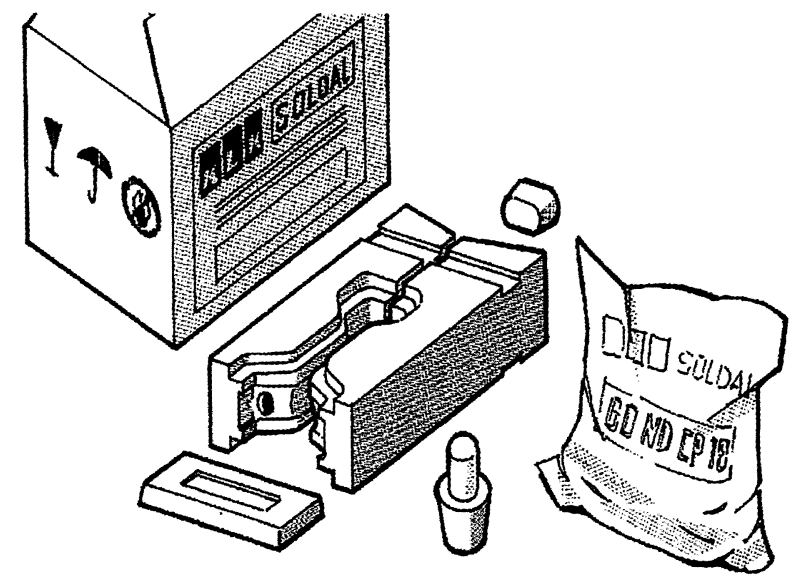

Figura 1. Kit de soldadura.

Figure 1. Kit of weld.

aluminio y ferroaleaciones. El proceso de elaboración, selección, pesado y mezcla de los componentes requiere gran precisión y pureza (contenidos en fósforo y azufre bajos) y distribución granulométrica acorde con la homogeneidad de composición física y química (contenidos en carbono, manganeso, silicio, aluminio, cromo, molibdeno y vanadio opcionales) exigidas al acero aluminotérmico. El proceso aluminotérmico de soldadura - verdadera miniacería, como se ha presentado en el apartado precedente- está basado en la reacción:

$$
\begin{gathered}
\text { Óxido de hierro }+ \text { Aluminio }+ \text { Aleantes }= \\
=\text { Acero }+ \text { Escoria }+ \text { Calor }
\end{gathered}
$$

La mezcla de granos citada se hace reaccionar en un crisol, provocando su ignición con una bengala, resultando, al cabo de pocos segundos, su transformación en acero fundido y escoria que flota. Este acero, que se encuentra, como dijimos, a temperatura superior a los $2.000^{\circ} \mathrm{C}$, se cuela automáticamente en el conjunto cerámico que contiene los extremos o puntas de los carriles entre los que se verifica su soldadura por fusión. El producto de la reacción, el acero aluminotérmico, deberá poseer una composición química, carbono equivalente $\mathrm{C}+\mathrm{Mn} / 6$, microestructura y durezas preestablecidos, acordes con el carril. Basta decir a este respecto, que deben encuadrarse en un intervalo de durezas de $\pm 20 \mathrm{HBS}$ cualesquiera sea el tipo de acero, perfil del carril y procedimiento de soldadura empleados. Tan estrictas características de composición química, dureza y resistencia mecánica exigidas al acero aluminotérmico, hacen necesarios el empleo de dispositivos de colada y geometría de moldes, particularmente idóneos, para 
evitar tanto los defectos físicos (rechupes, porosidades, grietas de contracción) como los químicos (micro y macrosegregaciones), herencias del proceso de solidificación. Su ausencia proporciona a las uniones aluminotérmicamente soldadas, calidades mecánicas superiores a las exigidas por los ferrocarriles.

El "precalentamiento" del metal base, el carril, resulta obligatorio, por estar fabricado con aceros de contenido en carbono equivalente superiores a $0,42 \%$, pues, de lo contrario, aparecerían fases de no equilibrio en las zonas afectadas por el calor y en el propio acero aluminotérmico (troostita, bainita, martensita, austenita retenida) (Figs. 2, 3 y 4), debido a una excesiva velocidad de enfriamiento, con el riesgo consiguiente de aparición de tensiones, deformaciones y grietas.

En la tabla II, se resumen las modalidades, calor y temperaturas de precalentamiento, adoptadas por la tecnología KLK-SOLDAL.

La calidad de las soldaduras resulta similar en los tres procedimientos al uso. Debe señalarse que el precalentamiento normal, $\mathrm{PN}$, exige mayor destreza en los operarios y equipos de calentamiento más lentos que en el proceso corto, al objeto de conseguir la temperatura de $880-900^{\circ} \mathrm{C}$ requerida. Por ello, la mayoría de los ferrocarriles prefieren la modalidad de precalentamiento corto, $\mathrm{PC}$ (salvo en la soldadura de carriles aleados $\mathrm{Cr}-\mathrm{Mo}$, donde el procedimiento $\mathrm{PN}$ se impone) en la que la fiabilidad del soldador, equipo de calentamiento y temperatura alcanzada no son tan críticas como en el proceso normal, dado que la mayor parte del precalentamiento de las puntas de los carriles correrá a cargo del propio acero aluminotérmico fundido y colado en moldes de especial diseño.

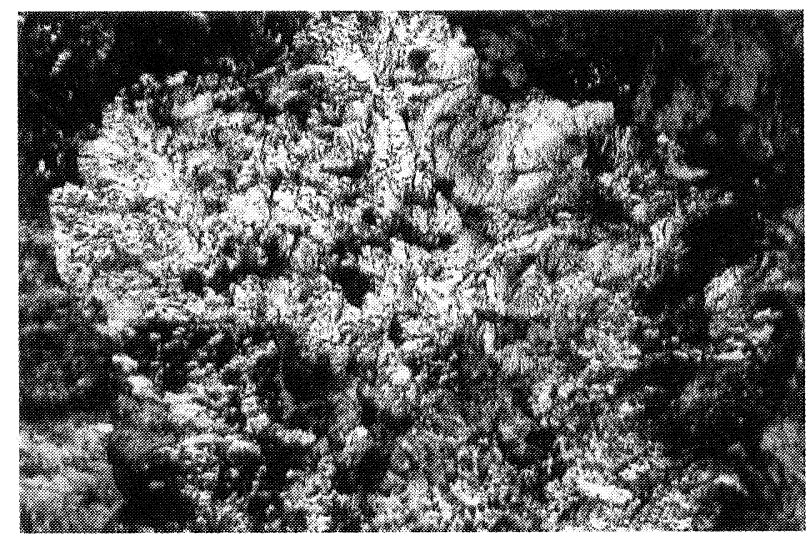

Figura 2. Acero aluminotérmico. Composición y precalentamiento correctos. Estructura perlítica (600X).

Figure 2. Aluminothermic steel. Correct composition and preheating (600X).

Rev. Metal. Madrid 40 (2004) 56-64

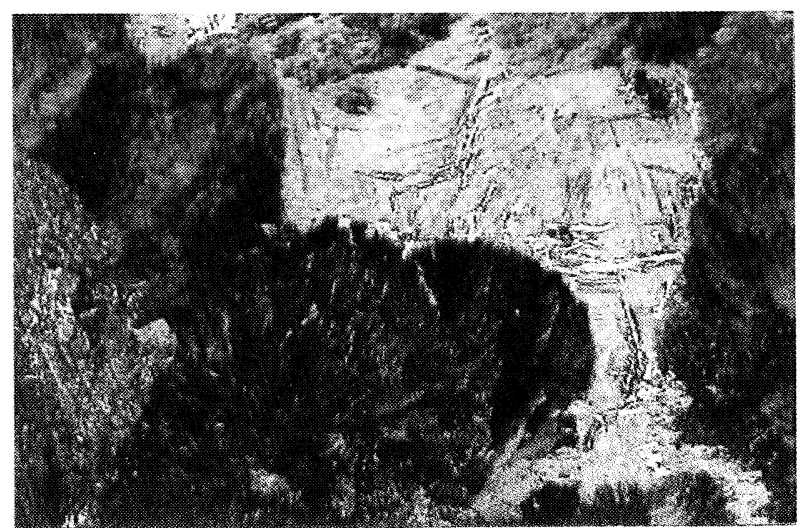

Figura 3. Acero aluminotérmico. Composición incorrecta. Precalentamiento correcto. Estructura troostito - bainítica (600X).

Figure 3. Aluminothermic steel. Incorrect composition. Correct preheating. Troostite-bainite structure (600X).

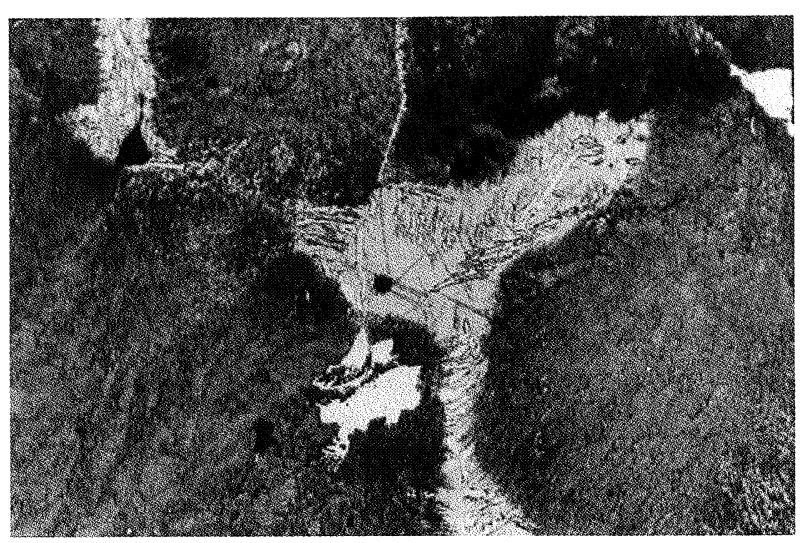

Figura 4. Acero aluminotérmico aleado. Composición correcta. Precalentamiento incorrecto. Islotes de martensita en la estructura perlítica (600X).

Figure 4. Aluminothermic alloyed steel. Correct composition. Incorrect preheating. Martensite pearlite structure (600X).

Los kits de soldadura para el precalentamiento corto, en calas de 24 y $48 \mathrm{~mm}$-calas corta y ancha, respectivamente- según tecnología KLKSOLDAL admiten las modalidades y equipos reseñados en la tabla III; además, esquemáticamente se apuntan, con carácter general, las ventajas e inconvenientes de cada sistema ${ }^{[7}$ y 8$]$.

\section{NORMAS RENFE-GIF SOBRE SOLDADURAS ALUMINOTÉRMICAS}

RENFE-GIF, al objeto de garantizar plenamente la calidad y fiabilidad de las soldaduras aluminotérmicas en vía, ha elaborado un conjunto de normas que pueden resumirse en cuatro apartados:

- Homologación de los procedimientos de soldadura. 
Tabla II. Diversos procedimientos para soldadura aluminotérmica

Table II. Different procedures for aluminothermic welding

\begin{tabular}{|c|c|c|c|c|c|c|}
\hline Procedimiento & Tipo & $\begin{array}{l}\text { Cala } \\
(\mathrm{mm})\end{array}$ & $\begin{array}{c}\text { Temperatura } \\
\text { precalentamiento } \\
\left({ }^{\circ} \mathrm{C}\right)\end{array}$ & $\begin{array}{c}\text { Sistemas } \\
\text { precalentamiento }\end{array}$ & $\begin{array}{l}\text { Tiempo } \\
\text { pto. (min) }\end{array}$ & Observaciones \\
\hline \multirow{3}{*}{ Precalentamiento normal } & PN & 14 & $850-900$ & & $6-7 / 10-18$ & Tecn. años 50 (obsoleta), no admite reparaciones \\
\hline & PN & $16 \pm 2$ & $850-900$ & -Oxi-propano & $6-7 / 10-18$ & Tecn. años 60 (obsoleta), no admite reparaciones \\
\hline & PN & $18 \pm 2$ & $850-900$ & -Aire-gasolina & $10-15$ & Tecn. años 70, no admite reparaciones \\
\hline Precalentamiento corto & PC & $23 \pm 2$ & $350-600$ & $\begin{array}{l}\text {-Propano } \\
\text {-Aire inducido }\end{array}$ & 6 & Tecn. actual años 90 , admite reparaciones \\
\hline $\begin{array}{l}\text { Precalentamiento corto. } \\
\text { Cala ancha }\end{array}$ & PA & $48 \pm 2$ & $350-600$ & $\begin{array}{l}\text {-Oxi-propano } \\
\text {-Aire-gasolina }\end{array}$ & $\begin{array}{l}1-2 \\
3-5\end{array}$ & $\begin{array}{l}\text { Tecn. actual años } 90 \text {, para reparaciones } \\
\text { Tecn. actual años } 90 \text {, para reparaciones }\end{array}$ \\
\hline
\end{tabular}

Tabla III. Diversos sistemas de precalentamiento corto. Ventajas e inconvenientes

Table III. Different systems of short preheating. Advantages and inconvenients

\begin{tabular}{|c|c|c|c|c|}
\hline Sistema & Presiones & Equipo & Ventajas & Inconvenientes \\
\hline $\begin{array}{l}\text { Propano } \\
\text { Aire inducido }\end{array}$ & $\begin{array}{c}\text { 2,5 bar } \\
\text { Atmosférica }\end{array}$ & $\begin{array}{l}\text { Botella propano, } 11 \mathrm{Kg} \\
+ \text { quemador } \mathrm{n}^{\circ} 1\end{array}$ & $\begin{array}{l}\text { Equipo barato y ligero } \\
\text { No se sobrepasan los } 500{ }^{\circ} \mathrm{C} \\
\text { Gran facilidad de manejo } \\
\text { Errores casi imposibles }\end{array}$ & Tiempo de precalentamiento $6 \mathrm{~min}$. \\
\hline $\begin{array}{l}\text { Propano } \\
\text { Oxígeno }\end{array}$ & $\begin{array}{l}1,5 \text { bar } \\
5 \text { bar }\end{array}$ & $\begin{array}{l}\text { Botella propano, } 35 \mathrm{Kg} \text {, } \\
\text { Botella oxígeno, } 10 \mathrm{~m}^{3} \\
+ \text { quemador } \mathrm{n}^{\circ} 2\end{array}$ & $\begin{array}{l}\text { Gran rapidez } \\
\text { Tiempo pto. } 1 / 2 \text { minutos }\end{array}$ & $\begin{array}{l}\text { Gran potencia calorífica } \\
\text { Si se utiliza mal puede fundir el carril }\end{array}$ \\
\hline $\begin{array}{l}\text { Aire } \\
\text { Gasolina }\end{array}$ & 0,2 bar & $\begin{array}{l}\text { Equipo motocompresor } \\
+ \text { quemador } n^{\circ} 3\end{array}$ & $\begin{array}{l}\text { En algunos lugares puede } \\
\text { ser difícil disponer de propano } \\
\text { Tiempo pto. } 3 / 5 \text { minutos }\end{array}$ & $\begin{array}{l}\text { Equipo más caro y pesado que los } \\
\text { anteriores }\end{array}$ \\
\hline
\end{tabular}

- Recepción en origen de los kits de soldadura.

- Formación y homologación de soldadores.

- Recepción de soldaduras en vías.

Las normas de homologación y recepción de procesos de soldadura aluminotérmica de carriles requieren, evidentemente, la necesaria adecuación del acero aluminotérmico con el acero base, en cierto modo, abierta a la preferencia y exigencias del usuario. Ensayos de recepción habituales son:

- Ensayos de dureza Brinell del acero aluminotérmico sobre la superficie de rodadura, con bola de $10 \mathrm{~mm}$ y carga de $3.000 \mathrm{Kg}$ (HBS 10/3000).

- Ensayos de dureza Vickers -efectuados sobre secciones longitudinales en la unión carrilcordón-carril, con carga de $30 \mathrm{Kg}$, a $5 \mathrm{~mm}$ de la

(c) Consejo Superior de Investigaciones Científicas

Licencia Creative Commons 3.0 España (by-nc) pista de rodadura. Pone de manifiesto la dureza del acero aluminotérmico, MA (metal de aporte), la anchura y dureza de las zonas afectadas por el calor, ZAC, -comprendida la zona "blanda" de perlita globular- y la calidad del metal base, MB (carril). Los perfiles adjuntos (Fig. 5) corresponden a soldaduras "aluminotérmicamente" correctas.

- Macroestructura de galletas longitudinales carril-cordón-carril. Permiten medir la anchura de las zonas de fusión -deberán ser superiores a 2,5 mm por cada lado-, zonas afectadas por el calor y zona reblandecida térmicamente (Fig. 6).

- Ensayo de flexión estática en tres puntos (con apoyo simple) aplicado en la parte central del cordón. Constituye el principal ensayo de caracterización mecánica de las soldaduras. En la 

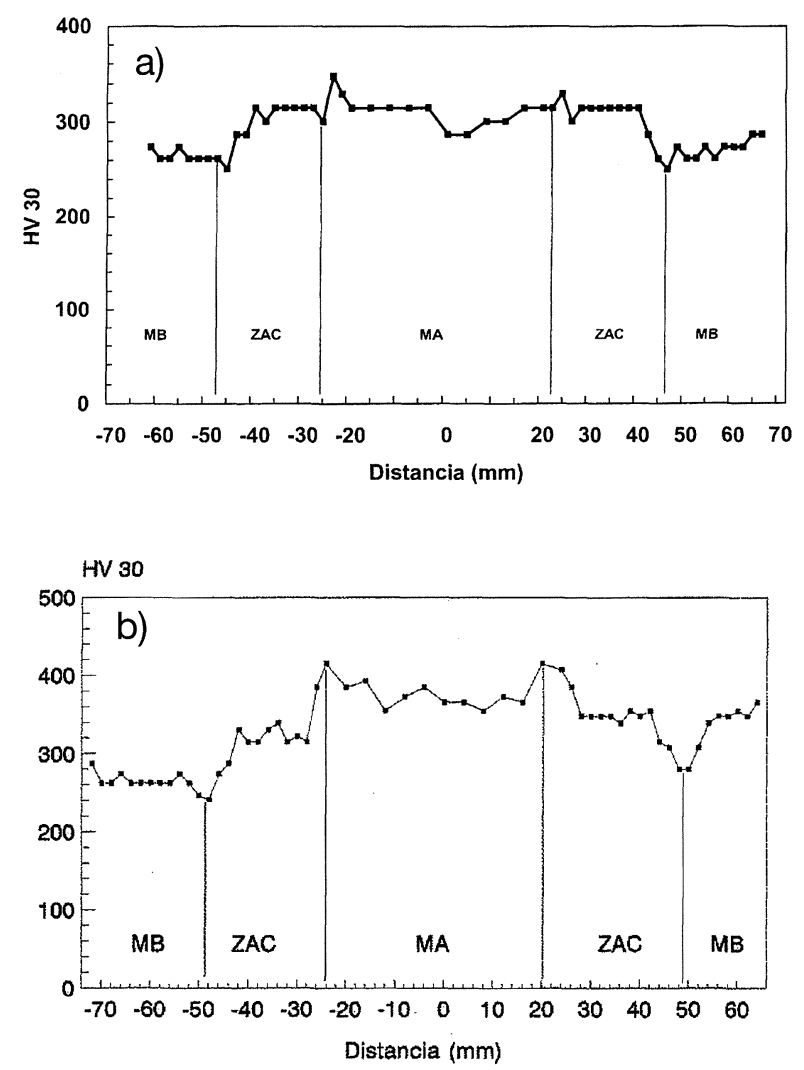

Figura 5. Perfil de durezas en soldadura de carriles:

a) UCl $60-900$

b) UCl $60-900 / \mathrm{UCl} 60-1100$.

Figure 5. Hardness profile of welded rails:

a) $\mathrm{UCl} 60-900$

b) UCl 60-900/ UCl 60-1100.

tabla IV y en la figura 7 se recogen los valores mínimos estipulados de resistencia, que fluctúa para soldaduras efectuadas en carriles: normal (700 MPa), duro (900 MPa) y extraduro (1.100 $\mathrm{MPa}$ ). En la práctica, las tensiones soportadas por la soldadura se equiparan o, en el peor de los casos, como mucho, resultan un $10 \%$ inferiores a la carga de rotura del carril -en cualquier caso superiores a los mínimos estipuladoslo que muestra la compacidad de la soldadura y ajuste del acero aluminotérmico ${ }^{[9}$ y 10$]$.

- Ensayo de flexión dinámica, en cuatro puntos, sobre apoyo bifurcado, que comprende la totalidad de la soldadura y zonas afectadas colindantes y duración de $2 \cdot 10^{6}$ ciclos y ciclado de carga, 200/20 MPa. El cupón soldado debe pasar el encargo y llevarse, posteriormente, a rotura en ensayo de flexión estática, con objeto de cuantificar el posible daño introducido por el proceso de fatiga al que se ha visto sometido el material (carril soldado): propagación de grietas a

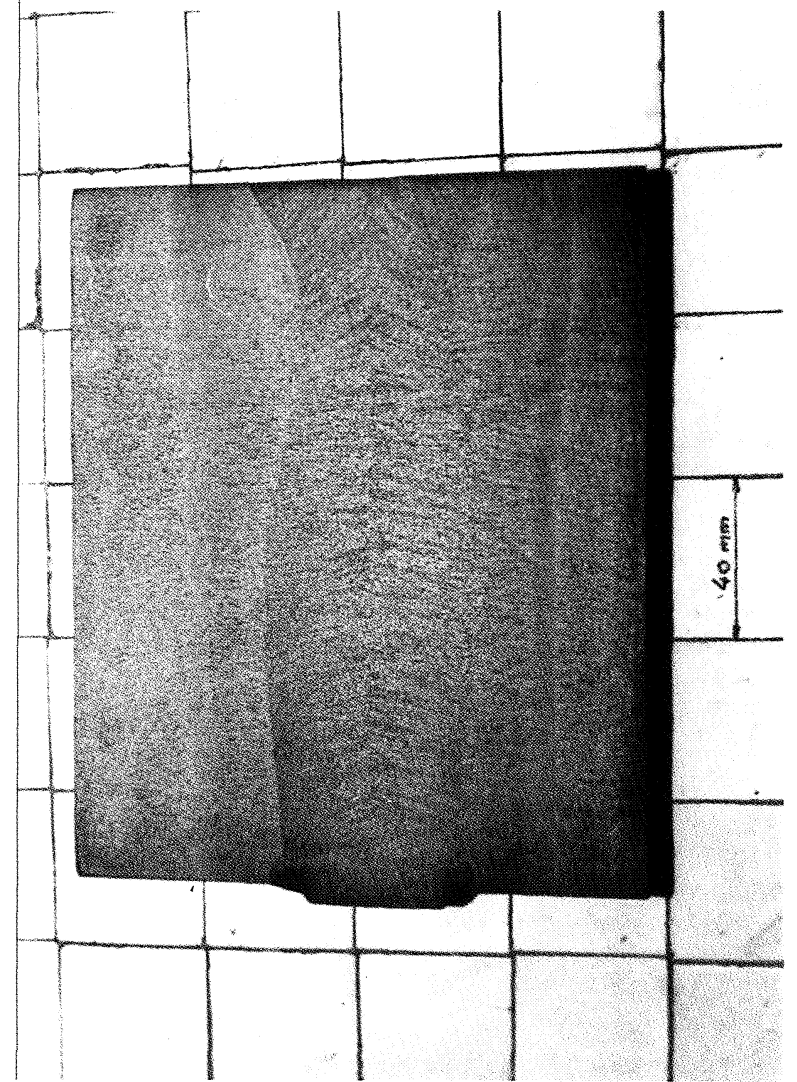

Figura 6. Macrografía de unión soldada mostrando zonas de fusión.

Figure 6. Macrography of welded joint showing the fusion zones.

Tabla IV. Resistencias mecánicas exigidas a carriles soldados

Table IV. Mechanical strengths demanded to welded rails

\begin{tabular}{|c|c|c|c|}
\hline Soldaduras Tipo & $700 \mathrm{MPa}$ & $900 \mathrm{MPa}$ & $1.100 \mathrm{MPa}$ \\
\hline Dureza del acero (HBS) & $260 \pm 20$ & $300 \pm 20$ & $340 \pm 20$ \\
\hline $\begin{array}{l}\text { Resistencia mínima en } \\
\text { flexión (MPa) }\end{array}$ & 500 & 600 & 700 \\
\hline $\begin{array}{l}\text { Flecha mínima en flexión } \\
(\mathrm{mm})\end{array}$ & 9 & 9 & 9 \\
\hline $\begin{array}{l}\text { Resistencia habitual en flexión } \\
\text { a } 9 \mathrm{~mm} \text { de flecha (MPa) }\end{array}$ & $600 / 650$ & $700 / 750$ & $850 / 900$ \\
\hline
\end{tabular}

partir de defectos preexistentes o zonas tensionadas, que abocan finalmente a la rotura de la pieza (Fig. 8). Por ser este ensayo particularmente sensible a los defectos geométricos o estructurales, en cierto modo, sintetiza con resultados obtenidos en los ensayos anteriormente mencionados. Solamente, juega en su contra un factor: el precio ${ }^{[11]}$. 


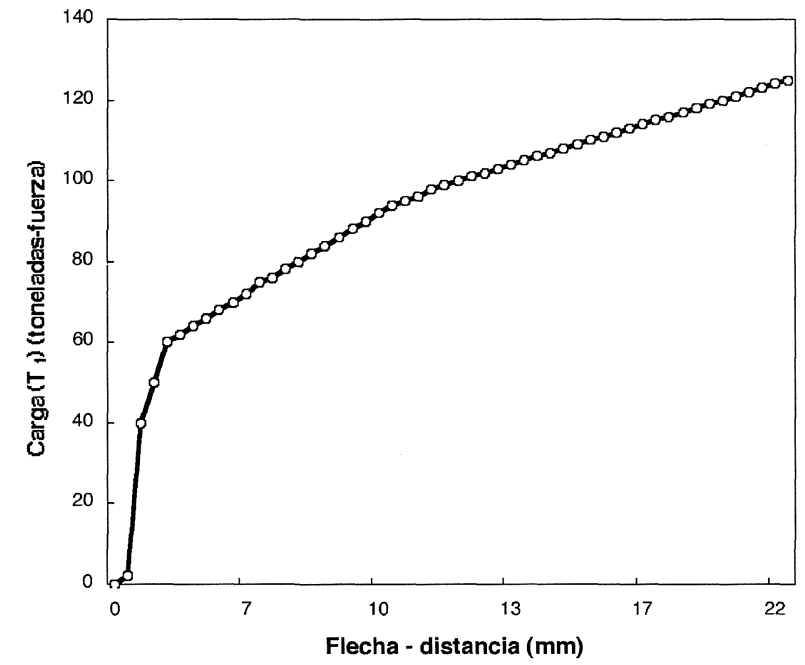

Figura 7. Diagrama esfuerzo aplicado, $T_{f}$ (toneladas-fuer$\mathrm{za}$ - flecha $(\mathrm{mm})$ en el ensayo de flexión estática de un carril soldado calidad $900 \mathrm{MPa}$.

Figure 7. Diagram of applied load, $T_{f}$ (ton-force) displacement $(\mathrm{mm})$ in the static bend test of a $900 \mathrm{MPa}$. welded quality rail.

\section{TENDENCIAS TECNOLÓGICAS. CONCLU- SIONES}

- En la actualidad, todos los ferrocarriles tienden a aumentar las cargas por eje, la velocidad, la frecuencia de paso, la dureza y la sección de los carriles. Se emplean carriles de mayor sección y más duros. Los tiempos de corte de vía para reparaciones son, cada vez, más reducidos.

- Se generaliza el uso de carriles aleados o tratados térmicamente (Fig. 9), con objeto de aumentar la durabilidad de las líneas. Los segundos son más fácilmente soldables que los primeros, al disminuirse los riesgos de aparición de zonas "estructuralmente" incorrectas y requerir, únicamente, precalentamientos cortos.

- Se tiende a eliminar el procedimiento de soldadura normal $\left(900^{\circ} \mathrm{C}\right), \mathrm{PN}$, y las calas reducidas $(14 \mathrm{~mm})$ en beneficio de los procesos de precalentamiento corto $\left(350^{\circ} \mathrm{C}\right)$ y calas de $23 \pm 2$ $\mathrm{mm}$. Es emergente la operación de soldadura con crisol de un solo uso.

- Las soldaduras aluminotérmicas, con los kits actualmente fabricados, se ejecutan con equipos sencillos y fáciles de manejar, garantizando zonas de fusión adecuadas, compacidad del acero aluminotérmico, estructuras perlíticas, resistencias a flexión cualificadas $(\mathrm{Rm}>750 \mathrm{MPa}$ para el perfil y calidad más común UIC 60 900), y límites de fatiga superiores a $200 \mathrm{MPa}$.
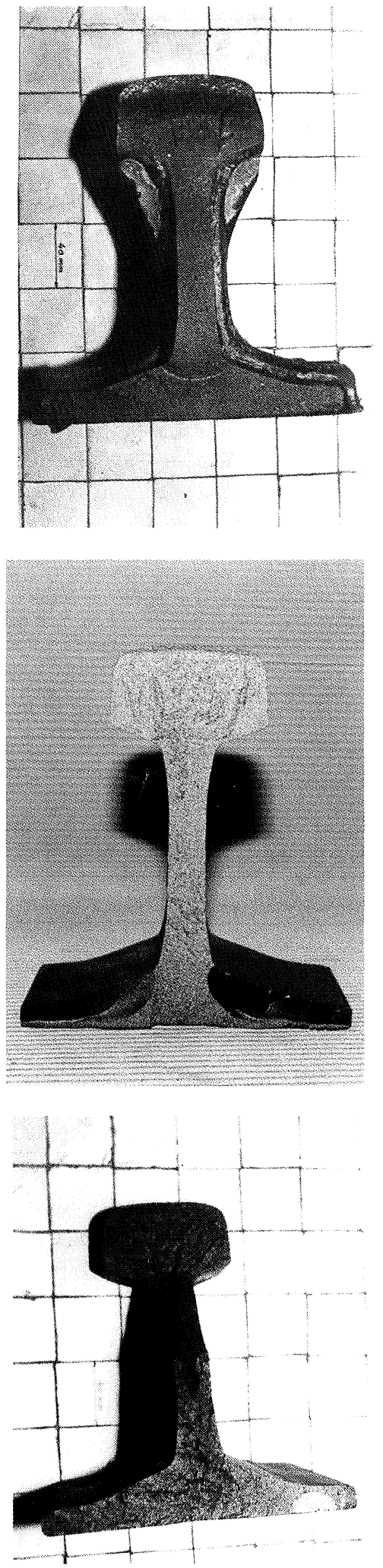

Figura 8. Rotura por fatiga:

a) En cabeza. Mancha oval.

b) En zona de acuerdo. Mancha elipsoidal.

c) En patín. Mancha en forma de "uña de dedo".

Figure 8. Break for fatigue:

a) In head. Oval mark.

b) In web-foot transition zone. Elliptical mark.

c) In foot. "Thumb nail" mark. 


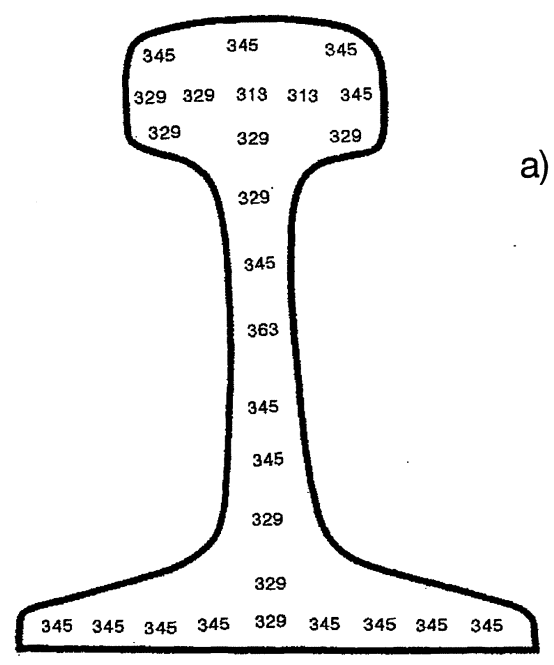

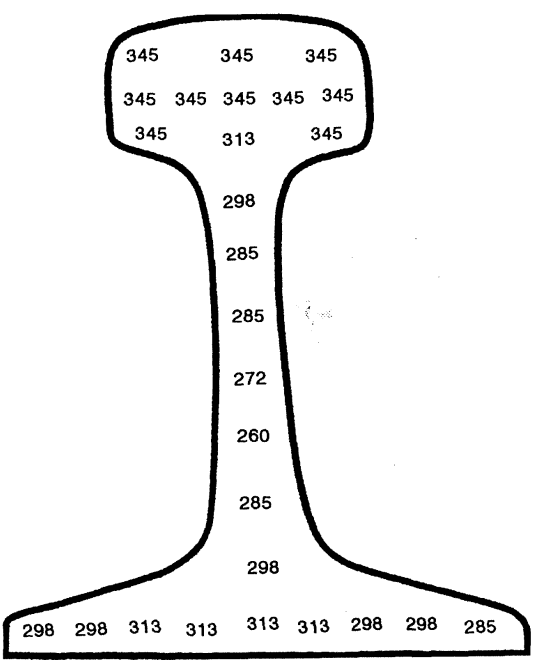

Figura 9. Carril tratado térmicamente. Mapa de durezas.

a) $0,80 \% \mathrm{C} ; 1,18 \% \mathrm{Mn} ; 0,17 \% \mathrm{Cr}$.

b) $0,78 \% \mathrm{C} ; 0,81 \% \mathrm{Mn}$.

c) $0,76 \% \mathrm{C} ; 1,24 \% \mathrm{Mn} ; 0,44 \% \mathrm{Cr} ; 0,12 \% \mathrm{Mo}$.

Figure 9. Heat treated rail. Hardness map.

a) $0,80 \% \mathrm{C} ; 1,18 \% \mathrm{Mn} ; 0,17 \% \mathrm{Cr}$.

b) $0,78 \% \mathrm{C} ; 0,81 \% \mathrm{Mn}$.

c) $0,76 \% \mathrm{C} ; 1,24 \% \mathrm{Mn} ; 0,44 \% \mathrm{Cr} ; 0,12 \% \mathrm{Mo}$

En conclusión, una soldadura correctamente ejecutada proporciona una soldadura de alta fiabilidad.

- No es fácil encontrar una PYME nacional que compita con éxito con empresas multinacionales superando los estándares requeridos por las normas europeas. Suministrar más detalles sobre el particular solo aportaría información a la competencia, que, por cierto, no suministra nada novedoso, a cambio, en sus artículos o Notas Técnicas.

\section{Agradecimientos}

Los autores quieren agradecer a D. Sergio Tamargo Fernández y D. Julio Rubio Cueli, Director General de KLK-ELECTROMATERIALES, por la colaboración y ayuda prestada en los procesos de soldadura aluminotérmica. Al Prof. José Ignacio Verdeja por sus comentarios en la redacción de la Nota Técnica. A D. José Ovidio García García y Doña Teresa Iglesias García su ayuda en la confección del trabajo experimental. Finalmente, al MCYT España por la financiación del MAT2003-00502. 


\section{REFERENCIAS}

[1] R. González, F. Carrillo y F.J. Vicario, Rev. Metal. Madrid 37 (2001) 653-664.

[2] R. Quintana, A. Cruz, L. Perdomo, G. Castellanos, L.L. García, A. Formoso y A. Cores, Rev. Metal. Madrid 39 (2003) 25-34.

[3] S. TAmargo, I Jornadas de Siderurgia de E.T.S.I.M.O., 2000, Oviedo, España.

[4] M. pastor, R. Sagaró y R. Cabrera, Rev. Metal. Madrid 37 (2001) 643-652.

[5] A. Ballester, L.F. VerdejA y J.P. SANCHO, Metalurgia extractiva. Fundamentos, Vol. I, Ed. Síntesis, Madrid, España, 2000, pp. 137-251.
[6] J.P. SAnChO, L.F. Verdeja y A. Ballester, Metalurgia extractiva. Procesos de obtención, Vol. II, Ed. Síntesis, Madrid, España, 2000, p. 30.

[7] J.I. Verdeja y J.A. Pero-Sanz, Revista de Minas Oviedo, 8 (1993) 103-108.

[8] J.I. Verdeja, J.O. García, D. Plaza y J.A. Pero-Sanz, Revista de Minas Oviedo, 15-16 (1997) 69-77.

[9] Norma RENFE P. R. V. 3-3-2.3. 1ª Edición, 1992, Madrid.

[10] Norma RENFE P. R. V. 3-3-2.4. 1 ${ }^{\text {a }}$ Edición, 1992, Madrid.

[11] J.I. Verdeja, J.O. García, D. Plaza y J.A. Pero-Sanz, Revista de Minas Oviedo 17-18 (1998) 77-82. 